\title{
Using delay to quench undesirable vibrations
}

\author{
M.K. Suchorsky $\cdot$ S.M. Sah $\cdot$ R.H. Rand
}

Received: 24 December 2009 / Accepted: 15 April 2010 / Published online: 6 May 2010

(C) Springer Science+Business Media B.V. 2010

\begin{abstract}
A van der Pol type system with delayed feedback is explored by employing the two variable expansion perturbation method. The perturbation scheme is based on choosing a critical value for the delay corresponding to a Hopf bifurcation in the unperturbed $\epsilon=0$ system. The resulting amplitude-delay relation predicts two Hopf bifurcation curves, such that in the region between these two curves oscillations will be quenched. The perturbation results are verified by comparison with numerical integration.
\end{abstract}

Keywords Differential-delay equations · Hopf bifurcation $\cdot$ Perturbation methods

\section{Introduction}

This paper concerns the effect of delay on the steady state behavior of systems described by differential

M.K. Suchorsky

Department of Mechanical and Aerospace Engineering, Cornell University, Ithaca, NY, USA

S.M. Sah

Faculty of Sciences Aïn Chock, University Hassan II-Aïn

Chock, BP 5366, Maârif, Casablanca, Morocco

R.H. Rand ( $\varangle)$

Department of Mathematics and Department of Mechanical and Aerospace Engineering, Cornell University, Ithaca, NY, USA

e-mail:rhr2@cornell.edu equations. The resulting equations, called differentialdelay equations (DDEs), may exhibit a diversity of steady state behaviors including stable equilibria and stable periodic motions. As an example, consider the following DDE which we investigate in this paper:

$$
\begin{aligned}
& \ddot{x}+x-\epsilon \gamma \dot{x}+\epsilon \alpha x^{2} \dot{x} \\
& \quad=\beta \dot{x}(t-T)+\delta x(t-T)
\end{aligned}
$$

This equation may be described as a version of van der Pol's equation with delay feedback. In what follows we will need to distinguish between delay amplitude and delay lag. The delay amplitudes associated with each of the terms on the right hand side of (1) are $\beta$ for the velocity feedback and $\delta$ for the position feedback. The delay lag is $T$ for both terms. We will show that a change in the steady state behavior may occur in response to a change in the delay amplitudes, the delay lag, and the other parameters of (1). In particular, periodic motions may appear or disappear due to Hopf bifurcations. Knowledge of such critical parameters can be used to quench or eliminate undesirable vibrations.

We begin with a review of some related literature. Considerable work has been done by other investigators on both single and coupled oscillators under delayed feedback. The literature is rich, with authors considering a wide variety of combinations of delay amplitudes and delay lags, and employing a range of 
perturbation techniques. The choice of which perturbation method to use is often related to the magnitude of the delay amplitude being considered. When the delay amplitude appears as $O(\epsilon)$, the method of averaging is a natural choice. Such was the approach taken in [1-3]. Atay [1] considered a van der Pol system under delayed position feedback, with both delay amplitude and delay lag taken as $O(\epsilon)$. The delay lag however need not be considered small in order to employ averaging, as Morrison and Rand [2] and Wirkus and Rand [3] both considered $O(1)$ delay lag in their work. Morrison and Rand [2] considered a delayed Mathieu equation, and Wirkus and Rand [3] considered two van der Pol oscillators with delay coupling. A system with delay amplitude of $O(\epsilon)$ and very large delay lag of $O(1 / \epsilon)$ was investigated by Das and Chatterjee [4] using multiple time scales instead of averaging. Systems where the delay amplitude is $O(1)$ have been considered by [5-8]. Numerous techniques have been employed in this case with Rand and Verdugo [5] using Lindtstedt's method, Maccari [6] using asymptotic perturbation, Nayfeh [7] using a center manifold reduction and finally Das and Chatterjee [8] using multiple time scales. Again, in considering $O(1)$ delay amplitude these authors have considered both $O(1)$ and $O(\epsilon)$ delay lag.

In this paper Hopf bifurcations in (1) are explored by using the two variable expansion method. The fixed points of the resulting slow flow give an expression for the limit cycle amplitude as a function of the delay and system parameters. From the slow flow equations we determine the stability of the limit cycle created in the Hopf bifurcation. We show that in a certain range of parameters, the delay lag may be chosen so as to quench the limit cycle via a Hopf bifurcation. In particular, the resulting amplitude-delay relation predicts two Hopf bifurcation curves, such that in the region between these two curves oscillations will be quenched. A similar system where both the delay amplitude and delay lag were $O(\epsilon)$ has been considered previously by Atay [1], who used the method of averaging. In comparison with our results, the results of [1] show a single Hopf bifurcation curve which corresponds to one of the two obtained by us. We compare our results with those of Atay [1] and with numerical integration.

\section{Perturbation analysis}

\subsection{Simple harmonic oscillator with delayed feedback}

This work considers a van der Pol type oscillator under delayed feedback:

$\ddot{x}+x-\epsilon \gamma \dot{x}+\epsilon \alpha x^{2} \dot{x}=\beta \dot{x_{d}}+\delta x_{d}$

The subscript $d$ denotes a delayed quantity, for example $x_{d}=x(t-T)$. We take the parameters $\epsilon, \gamma, \alpha, \beta$ and $\delta$ to be positive. When $\epsilon=0$, (2) becomes a linear oscillator with delayed feedback:

$\ddot{x}+x=\beta \dot{x_{d}}+\delta x_{d}$

Observe that when $\delta \geq 1$ in (3), we no longer have a harmonic oscillator for the case of zero delay lag. As the delay lag is increased from zero, the origin remains unstable and oscillations continue to be impossible. We therefore restrict $\delta<1$ in what follows. We introduce the parameter $T_{0}$, which will be the critical value of delay lag necessary for periodic solutions to (3) to exist.

$\ddot{x}+x=\beta \dot{x}\left(t-T_{0}\right)+\delta x\left(t-T_{0}\right)$

Looking for periodic solutions we assume solutions of the form,

$x=A \cos (\omega t)$

Substituting this assumed form into (4) yields a set of expressions that the system parameters must satisfy,

$$
\begin{aligned}
& \sin \left(\omega T_{0}\right)=-\frac{\beta \omega}{\omega^{2}-1} \\
& \cos \left(\omega T_{0}\right)=-\frac{\delta}{\omega^{2}-1}
\end{aligned}
$$

Squaring and adding (6) and (7) gives another form of these expressions,

$1=\frac{\left(\omega^{2}-1\right)^{2}}{\beta^{2} \omega^{2}+\delta^{2}} \Rightarrow \omega^{4}-\left(\beta^{2}+2\right) \omega^{2}+1-\delta^{2}=0$

Alternatively, dividing (6) by (7) yields,

$\tan \left(\omega T_{0}\right)=\frac{\beta \omega}{\delta}$ 
We can express $\omega^{2}$ in terms of $\beta$ and $\delta$ from (8).

$\omega^{2}=\frac{\beta^{2}+2}{2} \pm \sqrt{\left(\frac{\beta^{2}+2}{2}\right)^{2}-\left(1-\delta^{2}\right)}$

which can also be expressed as,

$\omega^{2}=\frac{\beta^{2}}{2}+1 \pm \sqrt{\left(\frac{\beta^{2}}{2}\right)^{2}+\beta^{2}+\delta^{2}}$

Since we consider $\delta<1$, both roots of $\omega^{2}$ will be positive and the assumed periodic solutions, (5), will exist. This can be seen from (10) by noting that the second term under the radical, $1-\delta^{2}$, will always be positive when $\delta<1$. This leads us to conclude the radical will always be less than the term preceding the radical. From (11) we observe that one root of $\omega$ will be less than 1 and the other greater than 1 . This is important as the quantity $\omega^{2}-1$ will play a role in determining the stability of the limit cycle through the slow flow equations.

The vanishing denominator in (6), (7) does not represent a singularity. This may be seen by noting that from (8), for $\omega$ to approach unity, both $\beta$ and $\delta$ must approach zero. The numerator and denominator of both (6), (7) thus simultaneously vanish. To show that the limit exists, one may use L'Hospital's Rule: Substituting $\omega$ from (10) into (6), (7) and taking the limit as $\beta$ goes to zero, where $\delta=c \beta$ for some constant $c$, turns out to give a nonsingular result.

In the next section we will perturb off of this solution to the $\epsilon=0$ system in order to find approximate solutions to (2) when $\epsilon \neq 0$.

\subsection{Two variable expansion method}

Returning to our original DDE, (2), we now perform a two variable expansion perturbation. Two variable expansion will produce slow flow equations which will capture the approach to the limit cycle, allowing us to determine its stability. Solving for the fixed points of the slow flow will give an amplitude-delay relation. For two variable expansion we define two time scales, a slow time scale, $\eta$, and a stretched time scale, $\xi$. The slower time scale will capture the approach to the periodic motion.

$\xi=\Omega t, \quad \eta=\epsilon t$
Since we are only working to $O(\epsilon)$, without loss of generality [9], we set the time stretch to be the frequency of the $\epsilon=0$ equation,

$\Omega=\omega+O\left(\epsilon^{2}\right)$

In terms of $\xi$ and $\eta$ our original DDE, (2), becomes,

$$
\begin{aligned}
& \Omega^{2} \frac{\partial^{2} x}{\partial \xi^{2}}+2 \Omega \epsilon \frac{\partial^{2} x}{\partial \xi \partial \eta}+\epsilon^{2} \frac{\partial^{2} x}{\partial \eta^{2}} \\
& +x+\left(-\epsilon \gamma+\epsilon \alpha x^{2}\right)\left(\Omega \frac{\partial x}{\partial \xi}+\epsilon \frac{\partial x}{\partial \eta}\right) \\
& =\beta\left(\Omega \frac{\partial x_{d}}{\partial \xi}+\epsilon \frac{\partial x_{d}}{\partial \eta}\right)+\delta x_{d}
\end{aligned}
$$

Next we expand $x, x_{d}$ in power series,

$$
\begin{aligned}
x(\xi, \eta)= & x_{0}(\xi, \eta)+\epsilon x_{1}(\xi, \eta)+\epsilon^{2} x_{2}(\xi, \eta)+\cdots \\
x_{d}(\xi, \eta)= & x\left(\xi_{d}, \eta_{d}\right) \\
= & x_{0}\left(\xi_{d}, \eta_{d}\right)+\epsilon x_{1}\left(\xi_{d}, \eta_{d}\right) \\
& +\epsilon^{2} x_{2}\left(\xi_{d}, \eta_{d}\right)+\cdots
\end{aligned}
$$

The delayed variables $\xi_{d}$ and $\eta_{d}$ are defined as,

$\xi_{d}=\Omega(t-T)$

$\eta_{d}=\epsilon(t-T)$

Recall that $T_{0}$ is the critical delay lag necessary for the $\epsilon=0$ system to undergo a Hopf bifurcation. Comparing the $\epsilon \neq 0$ system with the previous $\epsilon=0$ system, we see there is an additional linear term, $\epsilon \gamma \dot{x}$. Due to this additional linear term the critical value of delay lag necessary for a Hopf bifurcation in the $\epsilon=0$ system will be different than $T_{0}$. We anticipate that this additional linear term will cause only a small shift in the critical delay lag and accordingly choose to define the delay lag as,

$T=T_{0}+\epsilon \mu+O\left(\epsilon^{2}\right)$

With this definition of $T,(17)$ and (18) become,

$$
\begin{gathered}
\xi_{d}=\xi-\Omega\left(T_{0}+\epsilon \mu+O\left(\epsilon^{2}\right)\right) \\
\eta_{d}=\eta-\epsilon\left(T_{0}+\epsilon \mu+O\left(\epsilon^{2}\right)\right)
\end{gathered}
$$

Substituting (13) and neglecting terms of $O\left(\epsilon^{2}\right)$

$\xi_{d}=\xi-\omega T_{0}-\epsilon \omega \mu+\cdots$ 
$\eta_{d}=\eta-\epsilon T_{0}+\cdots$

We can now express $x_{d}$ in terms of $\xi-\omega T_{0}$ and $\eta$ by expanding it in a Taylor series about $\epsilon=0$. We neglect terms of $O\left(\epsilon^{2}\right)$.

$x_{d}(\xi, \eta)=x\left(\xi_{d}, \eta_{d}\right)$

$$
=\tilde{x}-\epsilon\left(\omega \mu \frac{\partial \tilde{x}}{\partial \xi}+T_{0} \frac{\partial \tilde{x}}{\partial \eta}\right)
$$

Where in this expression we have introduced $\tilde{x}$ for notational convenience, with $\tilde{x}$ defined by

$\tilde{x}=\tilde{x}(\xi, \eta)=x\left(\xi-\omega T_{0}, \eta\right)$

Substituting the power series expression for $x_{d}$, (16), into (24), and again neglecting terms of $O\left(\epsilon^{2}\right)$ we obtain a final expression for $x_{d}$.

$x_{d}(\xi, \eta)=\tilde{x}_{0}+\epsilon\left(\tilde{x}_{1}-\omega \mu \frac{\partial \tilde{x}_{0}}{\partial \xi}-T_{0} \frac{\partial \tilde{x}_{0}}{\partial \eta}\right)$

Substituting this final expression for $x_{d}$, (26), and $x$, (15), into the governing equation, (14), and collecting terms of $O(1)$ we recover a DDE similar to (4),

$\omega^{2} \frac{\partial^{2} x_{0}}{\partial \xi^{2}}+x_{0}-\delta \tilde{x_{0}}-\omega \beta \frac{\partial \tilde{x}_{0}}{d \xi}=0$

We define the left hand side of (27) to be $L\left(x_{0}\right)$.

$L\left(x_{0}\right)=\omega^{2} \frac{\partial^{2} x_{0}}{\partial \xi^{2}}+x_{0}-\delta \tilde{x_{0}}-\omega \beta \frac{\partial \tilde{x}_{0}}{d \xi}$

We take the general solution of (27) to be of the form

$x_{0}(\xi, \eta)=A(\eta) \cos (\xi)+B(\eta) \sin (\xi)$

Substituting this general solution into the $O(1) \mathrm{DDE}$, (27), yields a set of expressions that $\omega$ and $T_{0}$ must satisfy for given parameters $\beta$ and $\delta$.

$$
\begin{aligned}
& \sin \left(\omega T_{0}\right)=-\frac{\beta \omega}{\omega^{2}-1} \\
& \cos \left(\omega T_{0}\right)=-\frac{\delta}{\omega^{2}-1}
\end{aligned}
$$

These expressions are the same as those found in the previous section, cf. (6), (7). Again, these expressions can alternatively be expressed as (8), (9).

Returning to the step of substituting $x$ and $x_{d}$ into the governing equation (14), we now collect terms of $O(\epsilon)$.

$$
\begin{aligned}
& L\left(x_{1}\right) \\
& \qquad=-2 \omega \frac{\partial^{2} x_{0}}{\partial \xi \partial \eta}-\omega\left(\alpha x_{0}^{2}-\gamma\right) \frac{\partial x_{0}}{\partial \xi}-\omega^{2} \beta \mu \frac{\partial^{2} \tilde{x}_{0}}{\partial \xi^{2}} \\
& \quad-\omega \beta T_{0} \frac{\partial^{2} \tilde{x}_{0}}{\partial \xi \partial \eta}-\delta \omega \mu \frac{\partial \tilde{x}_{0}}{\partial \xi}+\left(\beta-\delta T_{0}\right) \frac{\partial \tilde{x}_{0}}{\partial \eta}
\end{aligned}
$$

Next we substitute (29) for $x_{0}$ into (32) and eliminate resonance by equating to zero the coefficients of $\cos (\xi)$ and $\sin (\xi)$. Doing so yields the slow flow equations on coefficients $A$ and $B$ of the general solution (29).

$\frac{d A}{d \eta}=\frac{G}{F}$
$\frac{d B}{d \eta}=\frac{H}{F}$

where

$$
\begin{aligned}
G= & -\omega_{0}\left(B \beta^{2} \omega_{0}^{2} \mathrm{~T}_{0}+B \delta^{2} \mathrm{~T}_{0}-2 A \omega_{0}^{3}\right. \\
& \left.+A \beta^{2} \omega_{0}+2 A \omega_{0}-B \beta \delta\right) \\
& \times\left(4 \omega_{0}^{2} \gamma-4 \gamma+4 \beta^{2} \mu \omega_{0}^{2}-\alpha B^{2} \omega_{0}^{2}\right. \\
& \left.-A^{2} \alpha \omega_{0}^{2}+4 \delta^{2} \mu+\alpha B^{2}+A^{2} \alpha\right) \\
H= & \omega_{0}\left(A \beta^{2} \omega_{0}^{2} \mathrm{~T}_{0}+A \delta^{2} \mathrm{~T}_{0}+2 B \omega_{0}^{3}\right. \\
& \left.-B \beta^{2} \omega_{0}-2 B \omega_{0}-A \beta \delta\right) \\
& \times\left(4 \omega_{0}^{2} \gamma-4 \gamma+4 \beta^{2} \mu \omega_{0}^{2}-\alpha B^{2} \omega_{0}^{2}\right. \\
& \left.-A^{2} \alpha \omega_{0}^{2}+4 \delta^{2} \mu+\alpha B^{2}+A^{2} \alpha\right) \\
F= & 4\left(T_{0}^{2}\left(\omega^{2}-1\right)^{4}+\left(\omega^{2}-1\right)^{2}\left(4 \omega^{2}+\beta^{2}\right.\right. \\
& \left.\left.-2 \beta \delta T_{0}\right)-4 \beta^{2} \omega^{2}\left(\omega^{2}-1\right)\right)
\end{aligned}
$$

Transforming to polar coordinates $R$ and $\psi$, where $A=R \cos \psi$ and $B=R \sin \psi$, we obtain the following simplified slow flow equations,

$$
\begin{aligned}
& x_{0}(\xi, \eta)=R(\eta) \cos (\xi-\psi(\eta)) \\
& \frac{d R}{d \eta}=\frac{D}{F} \\
& \frac{d \psi}{d \eta}=\frac{E}{F}
\end{aligned}
$$


where

$$
\begin{aligned}
D= & R \omega^{2}\left(\omega^{2}-1\right)\left(2\left(\omega^{2}-1\right)-\beta^{2}\right) \\
& \times\left(4 \gamma+4 \mu\left(\omega^{2}-1\right)-\alpha R^{2}\right) \\
E= & 4 T_{0} \mu \omega\left(\omega^{2}-1\right)^{4}+T_{0}\left(\omega^{2}-1\right)^{3}\left(4 \gamma \omega-\alpha R^{2}\right) \\
& -4 \beta \delta \mu \omega\left(\omega^{2}-1\right)^{2} \\
& +\beta \omega\left(\omega^{2}-1\right)\left(\alpha R^{2} \delta-4 \delta \gamma\right)
\end{aligned}
$$

A fixed point in the slow flow corresponds to a periodic motion in the original system. Solving for the fixed points of $\frac{d R}{d \eta}$ in (39) we obtain the amplitude of the periodic motion.

$$
R_{0}^{2}=\frac{4}{\alpha}\left(\gamma+\mu\left(\omega^{2}-1\right)\right)
$$

Plugging this amplitude expression into $\frac{d \psi}{d \eta}$, (40), for $R^{2}$ we find that the fixed point solutions to $\frac{d R}{d \eta}$, (39), identically satisfy $\frac{d \psi}{d \eta}$ meaning

$$
\frac{d \psi}{d \eta}=0
$$

and integration yields

$\psi=\psi_{0}$

This gives the final expression for the approximation to the periodic solution as

$$
x=x_{0}+O(\epsilon)=R_{0} \cos \left(\omega t-\psi_{0}+O\left(\epsilon^{2}\right)\right)
$$

\subsection{Stability of the limit cycle}

In addition to producing an approximation to the periodic solution, the slow flow equation on $R$, (39), can be used to determine the stability of the limit cycle. We begin by rewriting (39) in the form

$$
\frac{d R}{d \eta}=C_{1} R\left(4 C_{2}-\alpha R^{2}\right)
$$

where

$C_{1}=\frac{\omega^{2}\left(2\left(\omega^{2}-1\right)-\beta^{2}\right)}{4\left(T_{c r}^{2}\left(\omega^{2}-1\right)^{3}+\left(\omega^{2}-1\right)\left(4 \omega^{2}+\beta^{2}-2 \beta \delta T_{c r}\right)-4 \beta^{2} \omega^{2}\right)}$

$C_{2}=\gamma+\mu\left(\omega^{2}-1\right)$

Equation (47) represents a slow flow on the positive $R$-line $(R>0)$. It has two equilibrium points, $R=0$ and $R=\sqrt{4 C_{2} / \alpha}$, the latter of which corresponds to the limit cycle, cf. (43). The stability of limit cycle will therefore be opposite to that of the origin. In order for the limit cycle to exist, $C_{2} / \alpha>0$, and assuming $\alpha>0$, we must have $C_{2}>0$. The origin $R=0$ will be unstable if the product $C_{1} C_{2}>0$, and since $C_{2}>0$, the origin will be unstable (and the limit cycle will be stable) if $C_{1}>0$. Equation (48) can be rewritten in the form

$$
C_{1}=\frac{\omega^{2}\left(2-\frac{\beta^{2}}{\Delta}\right)}{4\left(T_{c r}^{2} \beta^{2} \omega^{2}+4 \omega^{2}\left(1-\frac{\beta^{2}}{\Delta}\right)+\left(\beta-\delta T_{c r}\right)^{2}\right)}
$$

where to get $C_{1}$ in this form, we have used (8) and have introduced the notation $\Delta=\omega^{2}-1$ for convenience.
We ask under what parameter conditions will $C_{1}>0$, which corresponds to a stable limit cycle. Note that the denominator is a sum of squares except for the $(1-$ $\left.\frac{\beta^{2}}{\Delta}\right)$ term. Also note that if $\left(1-\frac{\beta^{2}}{\Delta}\right)$ is positive then the numerator is also positive. Thus we may conclude that a sufficient condition for a stable limit cycle is

$\frac{\beta^{2}}{\Delta} \leq 1$

For every possible $\beta, \delta$ pair, where $0<\beta, 0<\delta<1$, (8) will return two values of $\omega$. One value will be less than 1 , while the other will be greater than 1 . For the case of $\omega<1, \Delta$ will be negative and inequality (51) is satisfied. For the case of $\omega>1, \Delta$ is positive and (51) is no longer immediately satisfied and additional work must be done to show that (51) still holds. From (30) we can write the following inequality,

$\frac{\beta^{2} \omega^{2}}{\Delta^{2}} \leq 1$ 
We can multiply this inequality, (52), by $\Delta$ without a sign change in the inequality. Multiplying (52) by $\Delta / \omega^{2}$ puts it in a form comparable to the inequality (51):

$\frac{\beta^{2}}{\Delta} \leq \frac{\Delta}{\omega^{2}}$

Expanding the right hand side of this inequality,

$\frac{\beta^{2}}{\Delta} \leq 1-\frac{1}{\omega^{2}}$

Since $\omega>1$ we can conclude,

$\frac{\beta^{2}}{\Delta} \leq 1-\frac{1}{\omega^{2}}<1$

This is the inequality (51) which we set out to show was true and therefore when $\omega>1$ we see that $C_{1}$ is positive. In summary we have shown that for both $\omega$ roots, $C_{1}$ will be positive which then implies that the limit cycle occurs when the origin is unstable and hence the limit cycle produced in the Hopf bifurcation will be attracting for all values of $\beta$ and $\delta$.

\section{Hopf bifurcation}

\subsection{Predicted Hopf bifurcation surface}

A supercritical Hopf bifurcation is characterized by a stable limit cycle born with zero amplitude, growing in size. The Hopf bifurcation occurs at the critical delay lag where the limit cycle has zero amplitude. We can use the amplitude-frequency perturbation result, (43), to predict the critical delay lag at which a Hopf bifurcation occurs. Setting $R_{0}=0$ we obtain the critical value of $\mu$ at which the Hopf bifurcation occurs.

$\mu_{c r}=\frac{\gamma}{\left(1-\omega^{2}\right)}$

Recall that $T_{0}$ is the delay lag at which a Hopf bifurcation occurs in the $\epsilon=0$ system. However, it turns out that $T_{0}$ will not be the delay lag necessary for a Hopf bifurcation in the $\epsilon>0$ system. The critical delay lag at which the $\epsilon>0$ system undergoes a Hopf bifurcation is given by (19), where $\mu$ equals $\mu_{c r}$ :

$T_{c r}=T_{0}+\epsilon \frac{\gamma}{\left(1-\omega^{2}\right)}+O\left(\epsilon^{2}\right)$

While this relation appears simple, in order to evaluate $T_{c r}$ we must first solve for $T_{0}$ and $\omega$. Recall that $\omega$ and $T_{0}$ respectively have polynomial and transcendental dependence on $\beta$ and $\delta$, (8) and (9). A closed form expression for $T_{c r}$ in terms of $\beta$ and $\delta$ is hence impossible. We can however create a $T_{c r}$ surface plot in Matlab for the delayed van der Pol case, (1). We also look at the curves resulting from three different cuts of this surface, $\beta=0, \delta=0$ and $\beta=\delta$.

Plotting $\omega$ vs. $\beta$ and $\delta$, verifies that there are two
Fig. 1 Surface plot of $\omega$ as a function of $\beta$ and $\delta$, as given by (8), with chosen parameters $\gamma=1, \alpha=1$

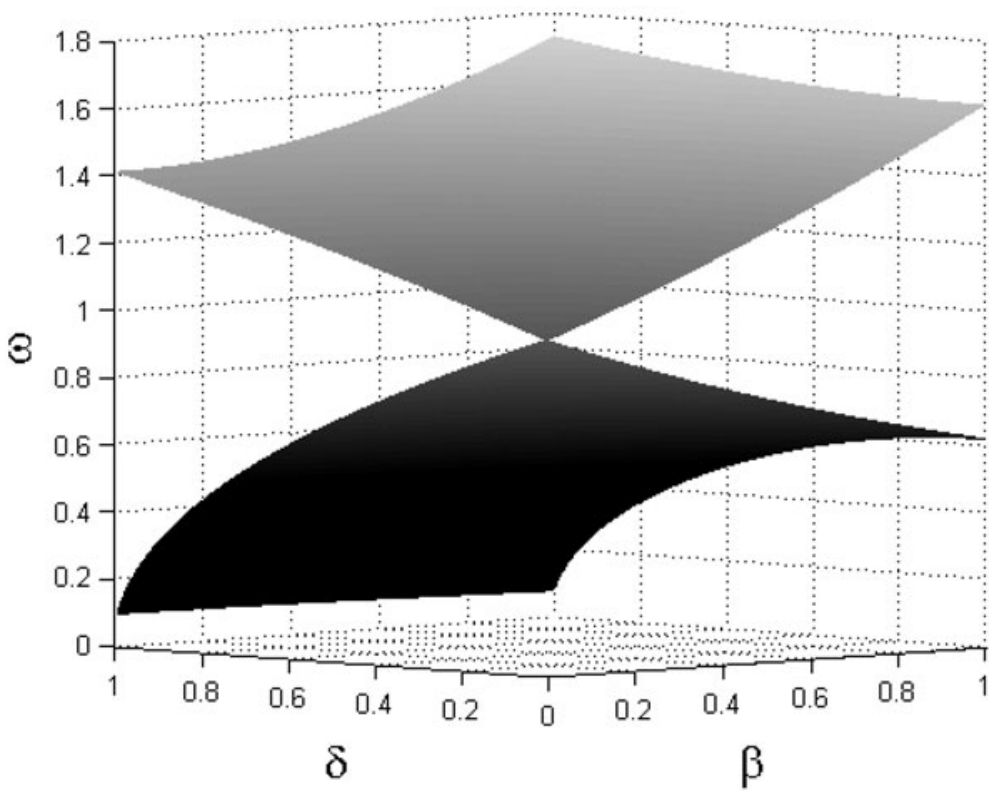


Fig. 2 Surface plot of the critical delay lag $T_{c r}$ necessary for a Hopf bifurcation as a function of $\beta$ and $\delta$, as given by (57), with chosen parameters $\gamma=1, \alpha=1, \epsilon=0.1$

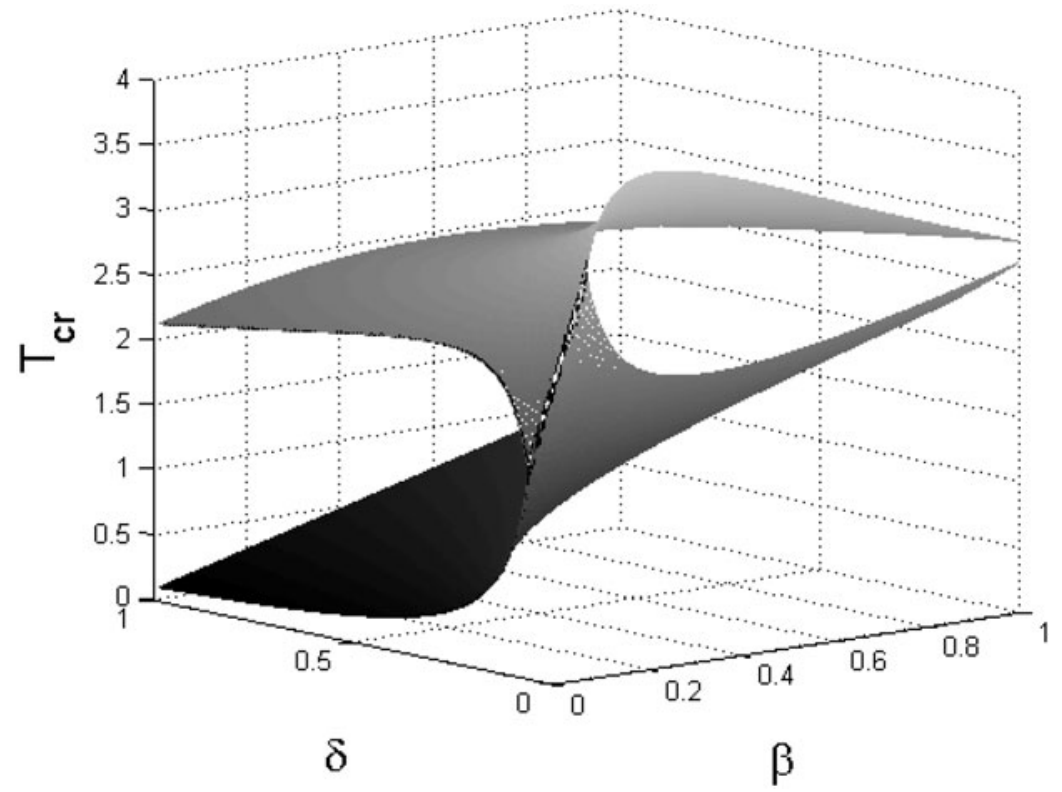

Fig. 3 Three different cuts of the Hopf bifurcation surface of Fig. 2, defined by (57), where $\gamma=1, \alpha=1$, $\epsilon=0.1$. Figure (a) shows the Hopf bifurcation curve produced by cutting the Hopf bifurcation surface at $\beta=0$. Similarly (b) and (c) show the Hopf bifurcation curves for $\delta=0$ and $\beta=\delta$ respectively
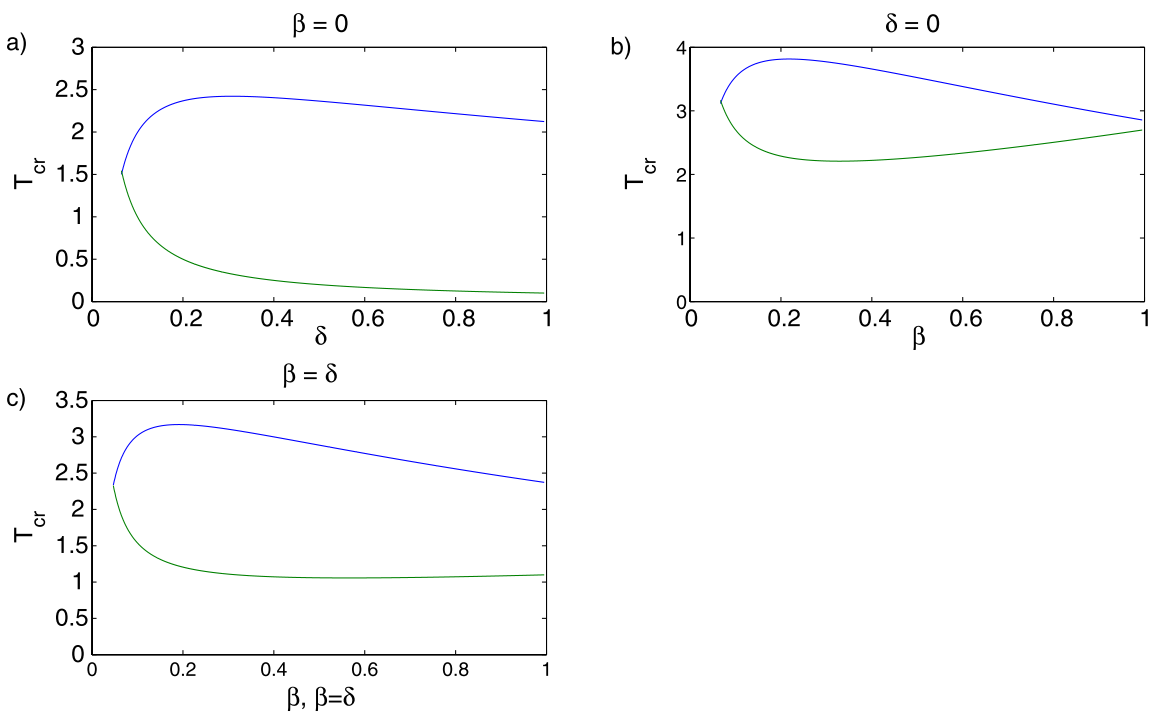

$\omega$ roots, one less than 1 and the other greater than 1 , Fig. 1. These two roots are contained on two distinct surfaces that meet in the single point $\omega=1$, as $\beta$ and $\delta$ are decreased to zero.

Figure 2 shows a plot of $T_{c r}$ vs. $\omega$ and $\beta$, and contains two surfaces, one surface for each of the two $\omega$ roots. The two $T_{c r}$ surfaces divide the parameter space into three distinct regions. Since points on these surfaces correspond to the occurrence of Hopf bifurcations, limit cycles are generically created or destroyed as these surfaces are crossed.
Recall that as $\beta$ and $\delta$ are decreased to zero $\omega$ goes to one. The effect of $\omega \rightarrow 1$ in (57) is for $T_{c r} \rightarrow \pm \infty$. The lower $T_{c r}$ surface in Fig. 2 goes to $\infty$ while the upper goes to $-\infty$. This causes the two surfaces to intersect at some values of $\beta$ and $\delta$ both larger than zero. The region beyond this intersection has been removed from the presented figures as it lies beyond the region of validity of the perturbation method.

In Fig. 3 we look at three different cuts of the $T_{c r}$ surface and confirm by numerical integration that the 
Fig. 4 Cut at $\beta=0$ of Hopf bifurcation surface, Fig. 3(a), defined by (57). The transition from a region of oscillation (a), to no oscillation (b), and back to oscillation (c), as the Hopf bifurcation curves are crossed, for $\gamma=1, \alpha=1$, $\epsilon=0.1$ is shown. Displayed is $x$ versus $t$ obtained by numerical integration of (2)
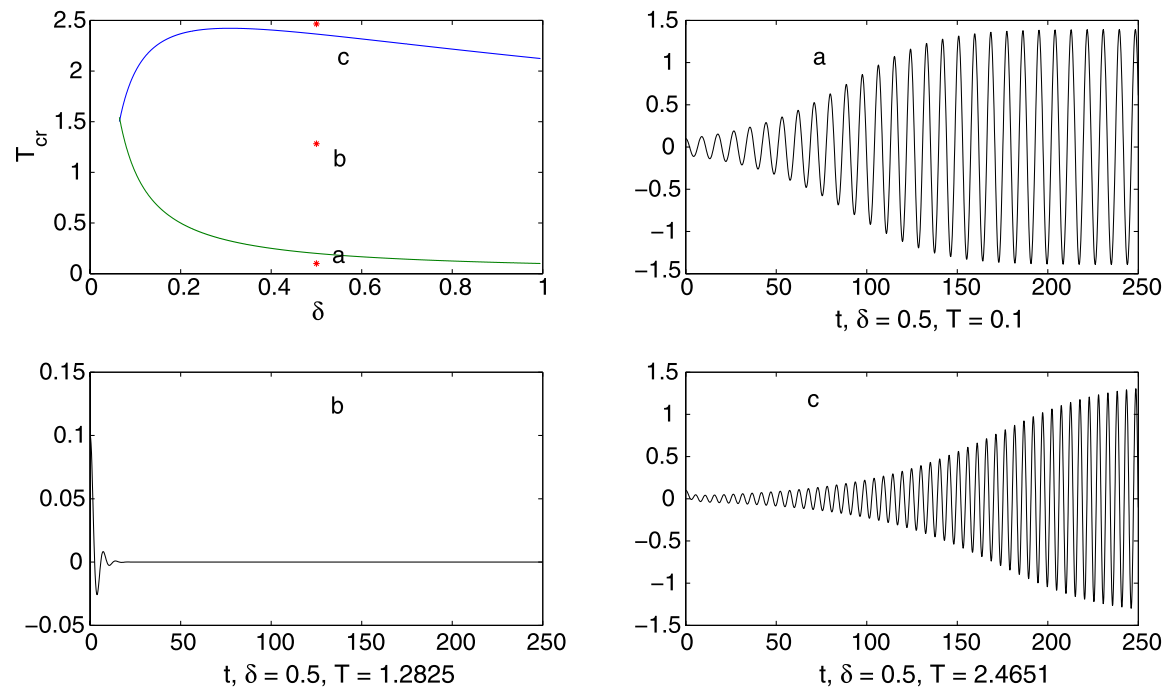

Fig. 5 Cut at $\delta=0$ of Hopf bifurcation surface,

Fig. 3(b), given by (57).

The transition from a region of oscillation (a), to no oscillation (b), and back to oscillation (c), as the Hopf bifurcation curves are crossed, for $\gamma=1, \alpha=1$, $\epsilon=0.1$ is shown. Displayed is $x$ versus $t$ obtained by numerical integration of (2)
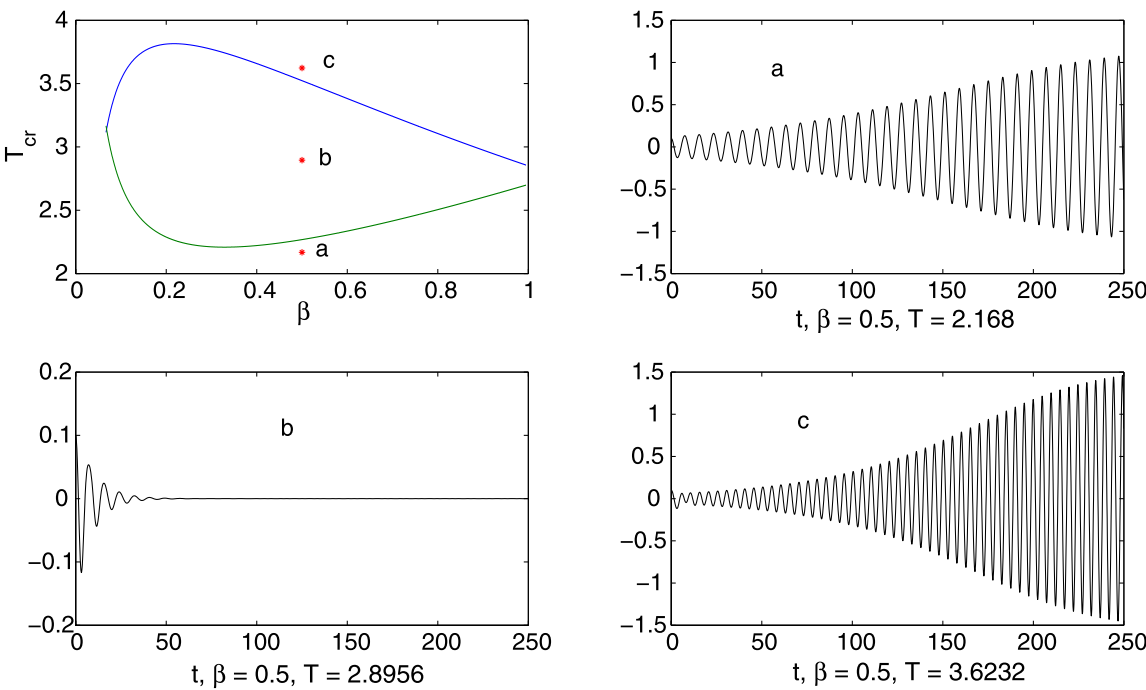

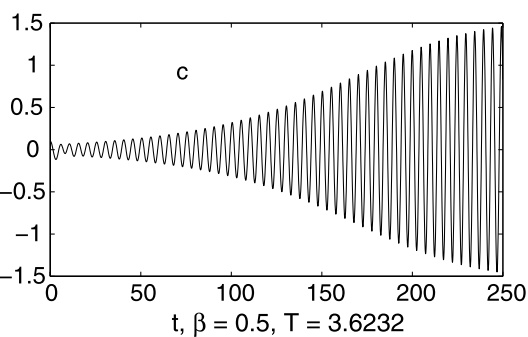

system transitions from oscillations to no oscillations and then back to oscillations as the delay lag is increased and the Hopf bifurcation curves are traversed. In Figs. 4-6 these transitions are shown by moving from point $a$ to $b$ to $c$. Each transition is accurately predicted by the derived Hopf bifurcation curves. We conclude that both small $(O(\epsilon))$, and large, $O(1)$ delay lag may be used as a means to quench a limit cycle.

Additionally we can create a surface plot of $T_{c r}$ as a function of $\epsilon$ and one of the delay amplitudes, $\delta$ or $\beta$, using (57). From (57) we see that $T_{c r}$ is linear in $\epsilon$, with parameters $T_{0}$ and $\omega$ varying only upon $\beta$ and $\delta$. We consider and plot the case of $\beta=\delta=0.5, \gamma=$ $\alpha=1$. The grey region in Fig. 7 represents no oscillations, and has been found using numerical integration. We can conclude that if $\epsilon$ is increased beyond some critical value, $\epsilon_{c r}$, the van der Pol terms become large and the limit cycle oscillation can no longer be quenched with delay.

\subsection{Comparison with Atay [1]}

Atay [1] considered the system,

$\ddot{x}+x-\epsilon\left(1-x^{2}\right) \dot{x}=\epsilon k x_{d}$ 
Fig. 6 Cut at $\beta=\delta$ of Hopf bifurcation surface, Fig. 3(c), given by (57). The transition from a region of oscillation (a), to no oscillation (b), and back to oscillation (c), as the Hopf bifurcation curves are crossed, for $\gamma=1, \delta=1$, $\epsilon=0.1$ is shown. Displayed is $x$ versus $t$ obtained by numerical integration of (2)
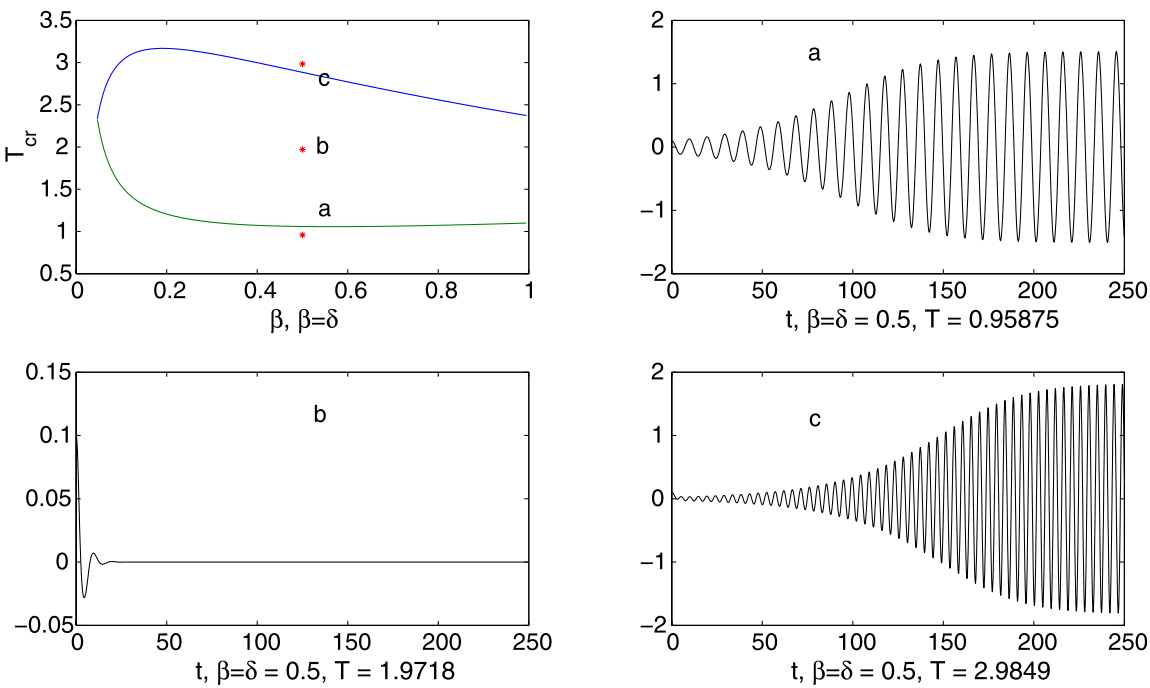

Averaging is a natural choice for a system with $O(\epsilon)$ delay amplitude terms. Atay [1] used the method of averaging to obtain the following amplitude expression,

$A m p^{2}=4(1-k \sin (T))$

Along with an $O(\epsilon)$ delay amplitude, Atay [1] also considered the delay lag to be small $(O(\epsilon))$. Our work is instead valid for both small and large delay lag and we considered the delay amplitudes to be $O(1)$. We compare the Hopf bifurcation curve predicted from our results with that predicted by [1] and also numerics. We expect our curve to be in better agreement with numerics than [1] when the delay lag is large and/or the delay amplitude is large. To compare our results with [1] we again consider the case of van der Pol oscillator with delay, setting $\gamma$ and $\alpha$ equal to one in our system (2). Additionally we need to equate our delay amplitudes with those of [1] implying, $\delta=\epsilon k$ and $\beta=0$. The Hopf bifurcation curves are plotted over $0<k<1 / \epsilon$. This range was chosen, recalling that $\delta<1$ for oscillation.

In Fig. 8 it is seen our results for small delay lag agree with Atay's. For large delay lag however the results diverge. This divergence grows when the delay lag is large and the delay amplitude $k$ is increased to also become large. As expected when $T_{c r}$ is large or when $k$ is large our results are in better agreement with numerics than the results in [1].

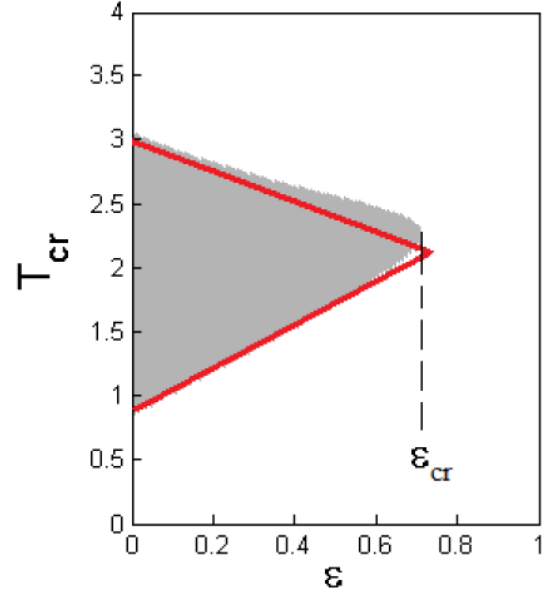

Fig. 7 Plot of $T_{c r}$ vs. $\epsilon$, as given by (57), where $\beta=\delta=0.5$. The grey region represents no oscillation, as concluded by numerical integration. Beyond $\epsilon_{c r}$ the oscillations may no longer be quenched with delay

\section{Conclusions}

We have studied a van der Pol type system under delayed feedback, (1), with delay amplitudes of $O(1)$ and delay lags not necessarily small. The two variable expansion perturbation scheme we used is based on choosing a critical value for the delay corresponding to a Hopf bifurcation in the unperturbed $\epsilon=0$ system. The perturbation method yielded two Hopf bifurcation curves, which were verified numerically. These two Hopf bifurcation curves bound a 
Fig. 8 Comparison of our Hopf bifurcation curve, (43), vs. Atay's [1], (59), which is based on assuming both small delay amplitude and small delay lag. $\times$ 's represent numerical integration of (2).

Parameters taken as $\gamma=1$, $\alpha=1, \beta=0, \epsilon=0.1$

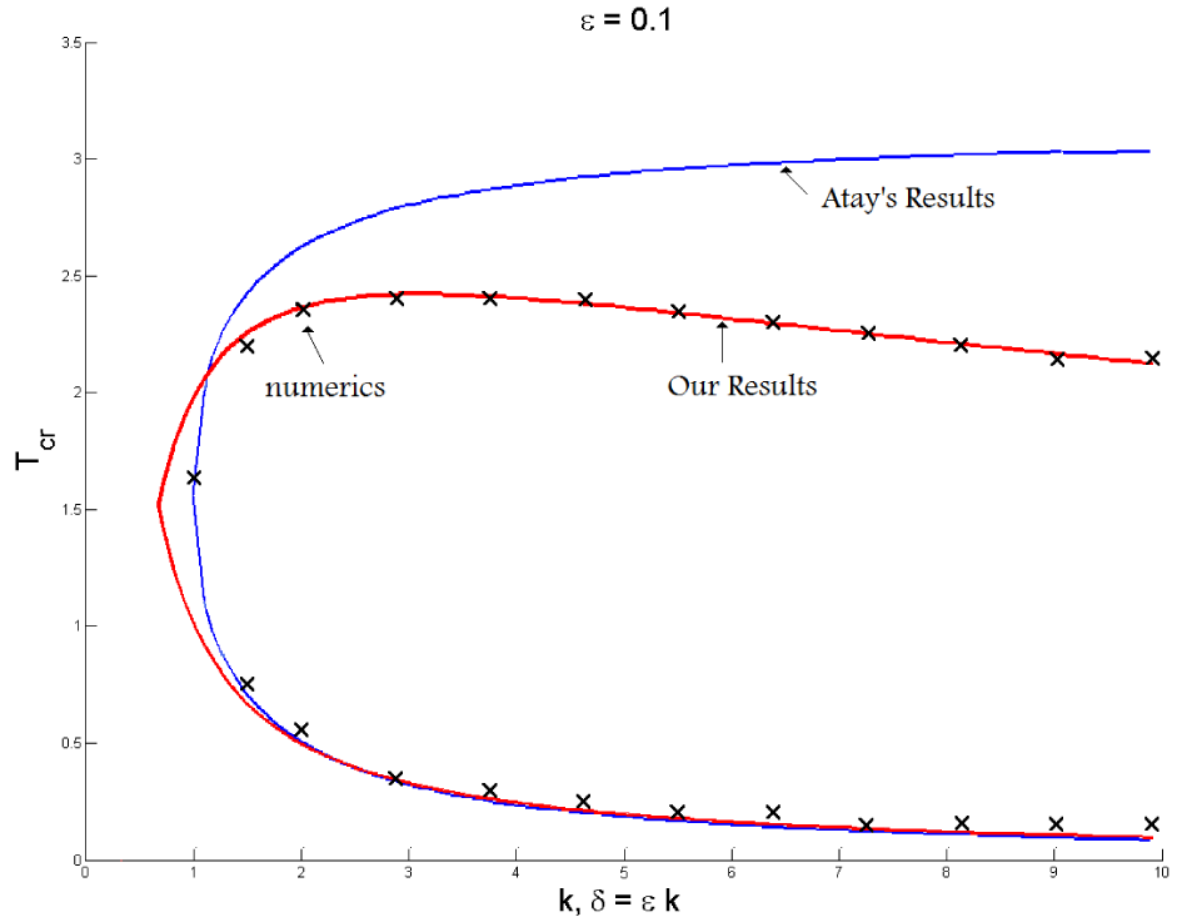

region of no oscillation, see Figs. 4-6. Oscillations may be quenched by appropriately varying the delay lag, so as to enter this region. We have therefore shown that delay may be used as a means to control and quench undesirable limit cycle oscillations.

Acknowledgements This work was partially supported under the CMS NSF grant CMS-0600174 "Nonlinear Dynamics of Coupled MEMS Oscillators". Support received from the Moroccan American Commission for Educational and Cultural Exchange through the Fulbright Program is acknowledged by author SMS. The hospitality of the Dept. of Theoretical and Applied Mechanics, Cornell University, is gratefully acknowledged.

\section{References}

1. Atay, F.M.: Van der Pol's oscillator under delayed feedback. J. Sound Vib. 218(2), 333-339 (1998)
2. Morrison, T.M., Rand, R.H.: 2:1 Resonance in the delayed nonlinear Mathieu equation. Nonlinear Dyn. 50, 341-352 (2007)

3. Wirkus, S., Rand, R.H.: The dynamics of two coupled van der Pol oscillators with delay coupling. Nonlinear Dyn. 30, 205-221 (2002)

4. Das, S.L., Chatterjee, A.: Second order multiple scales for oscillators with large delay. Nonlinear Dyn. 39, 375-394 (2005)

5. Rand, R.H., Verdugo, A.: Hopf bifurcation formula for first order differential-delay equations. Commun. Nonlinear Sci. Numer. Simul. 12, 859-864 (2007)

6. Maccari, A.: Vibration amplitude control for a van der PolDuffing oscillator with time delay. J. Sound Vib. 317, 20-29 (2008)

7. Nayfeh, A.H.: Order reduction of retarded nonlinear systems - the method of multiple scales versus centermanifold reduction. Nonlinear Dyn. 51, 483-500 (2008)

8. Das, S.L., Chaterjee, A.: Multiple scales without center manifold reductions for delay differential equations near Hopf bifurcations. Nonlinear Dyn. 30, 323-325 (2002)

9. Cole, J.D.: Perturbation Methods in Applied Math. Blaisdell, Boston (1968) 